Wright State University

CORE Scholar

$11-1-2000$

\title{
Combined Effects of Screening and Band Gap Renormalization on the Energy of Optical Transitions in $\mathrm{ZnO}$ and $\mathrm{GaN}$
}

D. C. Reynolds

David C. Look

Wright State University - Main Campus, david.look@wright.edu

B. Jogai

Follow this and additional works at: https://corescholar.libraries.wright.edu/physics

Part of the Physics Commons

\section{Repository Citation}

Reynolds, D. C., Look, D. C., \& Jogai, B. (2000). Combined Effects of Screening and Band Gap

Renormalization on the Energy of Optical Transitions in ZnO and GaN. Journal of Applied Physics, 88 (10), 5760-5763.

https://corescholar.libraries.wright.edu/physics/145

This Article is brought to you for free and open access by the Physics at CORE Scholar. It has been accepted for inclusion in Physics Faculty Publications by an authorized administrator of CORE Scholar. For more information, please contact library-corescholar@wright.edu. 


\title{
Combined effects of screening and band gap renormalization on the energy of optical transitions in ZnO and GaN
}

\author{
D. C. Reynolds, D. C. Look, and B. Jogai \\ Semiconductor Research Center, Wright State University, Dayton, Ohio 45435
}

(Received 3 July 2000; accepted for publication 31 August 2000)

\begin{abstract}
The energy positions of the optical transitions in both $\mathrm{GaN}$ and $\mathrm{ZnO}$ were investigated when the samples were excited simultaneously with a $\mathrm{HeCd}$ laser and $\mathrm{an}_{\mathrm{Ar}}{ }^{+}$ion laser. The increased number of free electrons excited by the $\mathrm{Ar}^{+}$ion laser will effectively screen both the free exciton and bound exciton transitions, resulting in a blueshift. The increased number of free electrons also produces many-body effects, which lead to a reduction of the band gap energy and thus a redshift. The resultant of screening and renormalization results in a redshift of the optical transitions in $\mathrm{ZnO}$ but a nearly vanishing shift in GaN. (C) 2000 American Institute of Physics. [S0021-8979(00)05123-9]
\end{abstract}

\section{INTRODUCTION}

Free-carrier screening will modify a spherical Coulomb potential, resulting in a reduced exciton binding energy. This will result in a blueshift of the optical transitions associated with both free excitons and bound excitons. The effects of free-carrier screening have been reported in both bulk materials such as $\mathrm{Ge}^{1-3}$ and GaSe (Ref. 4) and quantum-well structures. ${ }^{5-7}$ In this article we report on the position of the optical transitions in both $\mathrm{GaN}$ and $\mathrm{ZnO}$ crystals when they are excited simultaneously with two lasers. This experiment involves a HeCd laser at $3250 \AA$ which excites free electrons and holes, and an $\mathrm{Ar}^{+}$ion laser at $5145 \AA$, which excites electrons from the valence band to an intermediate level in the band and then from the intermediate level to the conduction band. The increased number of free electrons excited by the $\mathrm{Ar}^{+}$ion laser will effectively screen the free exciton as well as the bound exciton transitions. Screening results in a decrease in the binding energy of both types of transitions. In $\mathrm{ZnO}$, both the free- and bound-exciton transitions show a redshift as the exciting intensity of the $\mathrm{Ar}^{+}$ion laser in increased. The same transitions in GaN show essentially no energy shift with increased exciting intensity of the $\mathrm{Ar}^{+}$ion laser. The increased number of free electrons excited by the $\mathrm{Ar}^{+}$ion laser also produce many-body effects in intrinsic semiconductors, which lead to a renormalization of the band gap. The renormalization results in a redshift of the optical transitions due to reduction of the band gap energy. The energies of the optical transitions are then the resultant of the blueshift due to screening and the redshift due to renormalization.

We show that in both $\mathrm{GaN}$ and $\mathrm{ZnO}$, significant screening will occur at electron concentrations larger than 1 $\times 10^{16} \mathrm{~cm}^{-3}$ at $2 \mathrm{~K}$, the temperature of the experiment. We also show that the screening is weaker in $\mathrm{ZnO}$ than it is in $\mathrm{GaN}$. We further show that for the same electron concentration, the reduction in the band gap energy due to renormalization is greater in $\mathrm{ZnO}$ than in GaN. (From these results it is shown that a redshift in $\mathrm{ZnO}$ and a nearly vanishing energy shift in $\mathrm{GaN}$ is reasonable.) We also show that as excitons are screened from the donors, the donors are eliminated as decay paths for the excitons. The screened excitons may now decay as free excitons and thus increase the intensity of the free exciton transitions. In GaN, the free exciton associated with the $A$ valence band becomes the dominant optical transition, and the exciton associated with the $B$ valence band is also clearly observed. In $\mathrm{ZnO}$ both the $\Gamma_{5}$ and $\Gamma_{6}$ excitons associated with the $A$ valence band are well resolved and show a definite redshift. Screening is a very convenient way of enhancing the free exciton transitions.

\section{EXPERIMENTAL DETAILS}

The GaN sample was grown on a sapphire substrate by hydride vapor phase expitaxy to a thickness of $11 \mu \mathrm{m} .{ }^{8}$ The $\mathrm{ZnO}$ sample was grown by a seeded physical vapor transport method. ${ }^{9}$ In both cases, the $c$ axis of the crystal is normal to the growing surface. The final sample is bulk-like. Photoluminescence (PL) spectral measurements were made at $2 \mathrm{~K}$ with the sample immersed in liquid He. PL excitation was achieved with the $3250 \AA$ line of a HeCd laser. Additional electrons were excited in a two step process with the $5145 \AA$ line of an $\mathrm{Ar}^{+}$ion laser. The spectra were analyzed by means of a high-resolution $4 \mathrm{~m}$ grating spectrometer equipped with an RCAC31034A photomultiplier tube for detection.

\section{EXPERIMENTAL RESULTS}

The PL was excited with a $\mathrm{HeCd}(3250 \AA)$ laser, and in the screening process, the sample is simultaneously excited with an $\mathrm{Ar}^{+}$ion laser $(5145 \AA)$ that excites additional free electrons in a two step process involving deep impurities or defects. The steady-state concentration of additional electrons cannot be calculated since the cross sections for the various transitions are not known. An estimate of the number of electrons participating in the screening process can be made if one assumes that substantial screening will occur when the Debye length and the Bohr radius are comparable. Free carrier screening modifies the Coulomb potential $\phi$ $\sim e / 4 \pi \in r$ by adding a multiplicative factor, $\exp (-r / \lambda)$, where $r$ is the distance from the center of the charge, and $\lambda$ the Debye length given by ${ }^{10}$ 


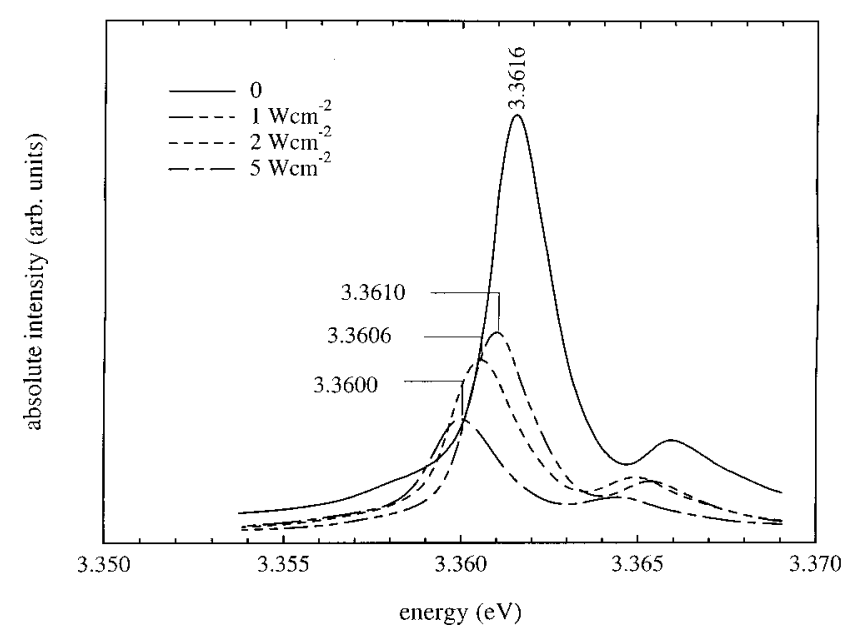

FIG. 1. Intensity and energy position of the $D,{ }^{0} \mathrm{X}$ transitions in $\mathrm{ZnO}$ as a function of the $\mathrm{Ar}^{+}$ion laser intensity.

$$
\lambda=\left(\varepsilon k T / e^{2} n_{\mathrm{eff}}\right)^{1 / 2} .
$$

The major part of the charge cloud will be at the Bohr radius $\alpha_{B}=0.529 \varepsilon / m^{*}$. In $\mathrm{GaN} m^{*} \simeq 0.22$ and $\varepsilon \simeq 9.5{ }^{11}$ giving a Bohr radius of $\simeq 22 \AA$. The Debye length will be comparable when $n_{\mathrm{eff}} \simeq 1 \times 10^{16} \mathrm{~cm}^{-3}$. In $\mathrm{ZnO}$ the dielectric constant is smaller, 8.12 vs 9.5 , and the mass is larger, 0.318 vs 0.22 , giving a smaller Debye length as well as a smaller Bohr radius. Therefore, roughly the same number of free electrons will produce substantial screening in $\mathrm{ZnO}$ as well.

Having an estimate of the number of free electrons required for the screening process, one can estimate the screened donor binding energy from the common empirical relationship used to describe donor screening

$$
E_{D}=E_{D 0}-\alpha N_{D}^{1 / 3},
$$

where $E_{D}$ is the screened donor binding energy, $E_{D 0}$ is the unscreened donor binding energy, and the suggested value of $\alpha$ for $n$-type $\mathrm{GaN}$ is $\alpha \simeq 2.1 \times 10^{-5} \mathrm{meV} \mathrm{cm} .{ }^{12}$ For $\mathrm{ZnO}$ we can approximate $\alpha$ as $\left[\left(\varepsilon_{\mathrm{ZnO}} / \varepsilon_{\mathrm{GaN}}\right)\left(m_{\mathrm{GaN}}^{*} / m_{\mathrm{ZnO}}^{*}\right)\right] \alpha_{\mathrm{GaN}}$ giving a value $\alpha \simeq 1.2 \times 10^{-5} \mathrm{meV} \mathrm{cm}$. One sees that the screening in $\mathrm{ZnO}$ is somewhat weaker than that in $\mathrm{GaN}$. Assuming $1 \times 10^{16} \mathrm{~cm}^{-3}$ free electrons, one calculates from Eq. (2) a reduction in the donor binding energy in $\mathrm{ZnO}$ of $\simeq 2.7 \mathrm{meV}$ due to screening. From screening alone, this should result in a blueshift of the donor-bound-exciton $\left(D^{0}, X\right)$ transition.

The transition energy of $D^{0}, X$ is the free exciton energy minus the energy with which the exciton is bound to the donor. By Haynes rule ${ }^{13}$ the exciton is bound to the donor by some fraction of the donor binding energy. It would be expected that the free exciton binding energy will be screened very similar to that of the donor binding energy. Therefore, in the screened case, the $D^{0}, X$ transition energy will be that of the screened free exciton energy minus the energy with which the exciton is bound to the screened donor. A reasonable estimate of the blue energy shift of $D^{0}, X$ with screening would then be obtained from Eq. (2). The $D^{0}, X$ PL spectra as a function of $\mathrm{Ar}^{+}$ion laser intensity are shown in Fig. 1. The $D^{0}, X$ intensity decreases as the $\mathrm{Ar}^{+}$ion intensity increases, as expected. As the excitons are screened from the

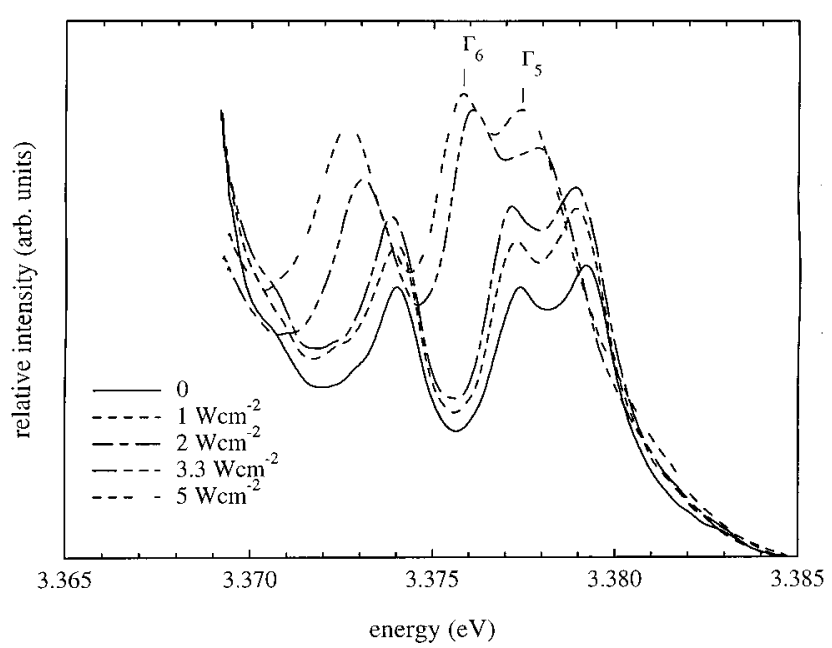

FIG. 2. Intensity and energy of the free exciton transitions in $\mathrm{ZnO}$ as a function of the $\mathrm{Ar}^{+}$ion laser intensity.

donors, the $D^{0}, X$ decay route is eliminated as one of the processes by which excitons are dissipated. Therefore, as more excitons are screened from the donors, the $D^{0}, X$ intensity will decrease and the free exciton intensity will increase. This is verified in Fig. 2 where the free exciton intensity is plotted as a function of the $\mathrm{Ar}^{+}$ion laser intensity. Here the $\Gamma_{5}$ and $\Gamma_{6}$ free excitons are clearly identified. One other feature is observed in both the $D^{0}, X$ spectra and the free exciton spectra and that is the energy redshift. This feature will be addressed following the presentation of the GaN exciton spectra.

The GaN sample was excited in exactly the same way as the $\mathrm{ZnO}$ sample. Making the assumption of $1 \times 10^{16} \mathrm{~cm}^{-3}$ free electrons for GaN, then from Eq. (2) a reduction in the donor binding energy of $\simeq 4.5 \mathrm{meV}$ due to screening is obtained. The PL emission spectra from $\mathrm{GaN}$ as a function of $\mathrm{Ar}^{+}$ion laser intensity are shown in Fig. 3. The $D^{0}, X$ emission peak $(3.4809 \mathrm{eV})$ as well as the ionized-donor-boundexciton $\left(D^{+}, X\right)$ peak $(3.4762 \mathrm{eV})$ are dominant when the $\mathrm{Ar}^{+}$ion laser intensity is zero. As the $\mathrm{Ar}^{+}$intensity is in-

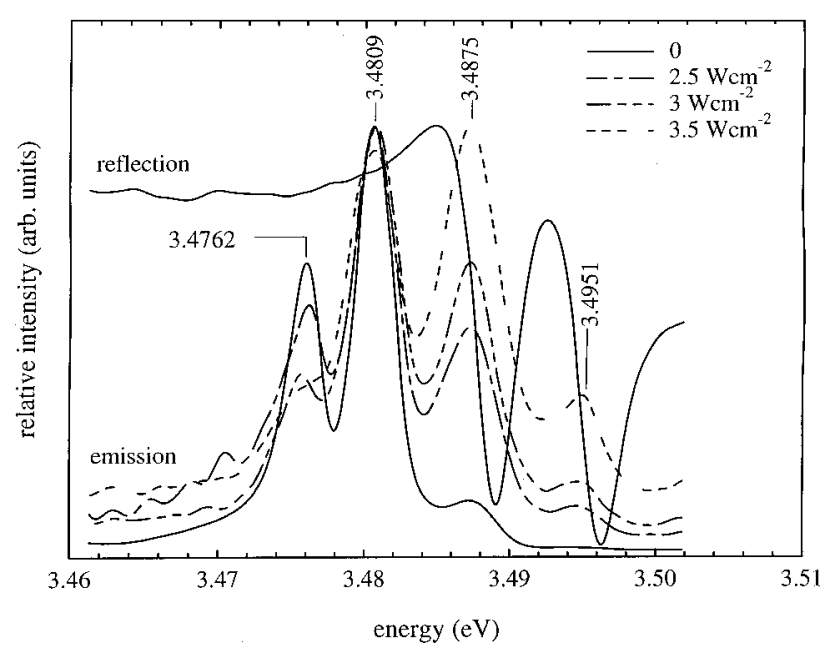

FIG. 3. The PL emission spectra from GaN as a function of the $\mathrm{Ar}^{+}$ion laser intensity. 


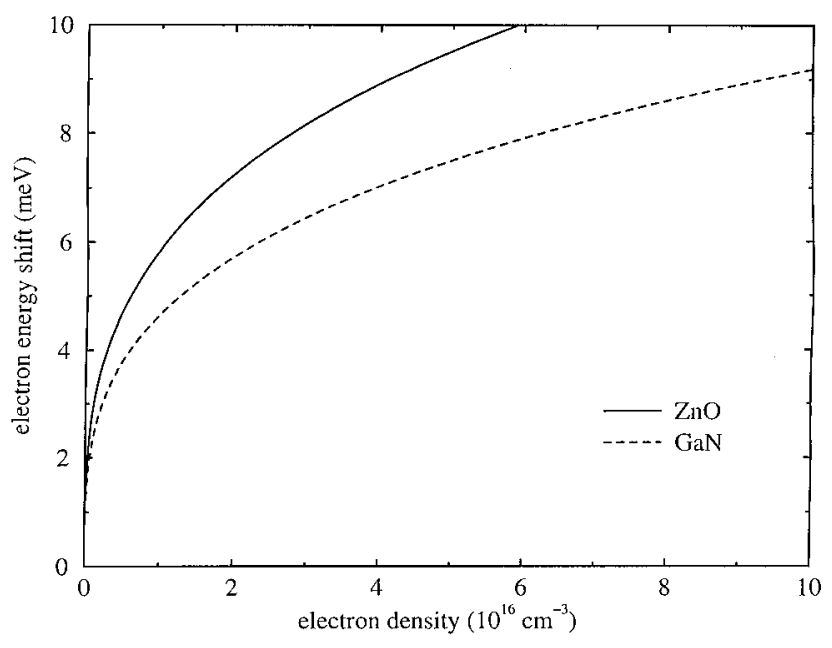

FIG. 4. The reduction of the renormalized energy gap as a function of electron density for both $\mathrm{ZnO}$ and $\mathrm{GaN}$.

creased, more free electrons that neutralize the ionized donors are created, and at the highest $\mathrm{Ar}^{+}$intensity the ionized donor excitons are essentially all neutralized. The $D^{0}, X$ centers experience an increase in numbers as the ionized donors are neutralized, but they also experience a decrease due to screening. At the highest intensity of the $\mathrm{Ar}^{+}$ion laser the free exciton associated with the $A$-valence band $(3.4875 \mathrm{eV})$ is the dominant transition and the free exciton associated with the $B$ valence band $(3.4951 \mathrm{eV})$ is clearly evident. In $\mathrm{GaN}$, as in $\mathrm{ZnO}$, the bound excitons decrease in intensity while the free excitons increase in intensity with an increase in $\mathrm{Ar}^{+}$ion laser intensity. The top solid line in Fig. 3 shows the reflection minima, which are associated with the $A$ - and $B$-band free excitons. In the case of $\mathrm{GaN}$, the bound and free excitons do not show an obvious blue- or redshift as the $\mathrm{Ar}^{+}$ ion laser intensity is increased.

The additional electrons excited by the $\mathrm{Ar}^{+}$ion laser may also result in many-body effects in an intrinsic semiconductor, which will lead to a renormalization of the fundamental energy gap. The renormalization reduces the energy gap as shown in Fig. 4. In this figure, the energy shift is estimated from density functional theory within the local density approximation using the Hedin Lundquist parametrization. ${ }^{14}$ Other parametrized forms exist and yield similar results. ${ }^{15}$ The reduction of the renormalized energy gap, as a function of electron density, is shown for both $\mathrm{GaN}$ and $\mathrm{ZnO}$ in Fig. 4. Here it is seen that renormalization reduces the $\mathrm{ZnO}$ band gap more than the $\mathrm{GaN}$ band gap for the same electron density. The PL energy positions are determined by a combination of renormalization and screening, the former giving a redshift and the latter an offsetting blueshift. For a given electron density, the blueshift can be obtained from Eq. (2) and the redshift from the graphs in Fig. 4. Taking electron densities within an order of magnitude of those calculated to give substantial screening, the blue- and redshifts for both $\mathrm{ZnO}$ and $\mathrm{GaN}$ are given in Table I. From Table I, one would expect to observe an increasing redshift of the exciton transitions from $\mathrm{ZnO}$ as the $\mathrm{Ar}^{+}$ion laser intensity is increased. In the case of GaN, essentially no shift
TABLE I. The blue and red energy shifts of the optical transitions in both $\mathrm{ZnO}$ and $\mathrm{GaN}$ as the electron density is increased.

\begin{tabular}{cccc}
\hline \hline Sample & $\begin{array}{c}\text { Electron } \\
\text { density }\left(\mathrm{cm}^{-3}\right)\end{array}$ & $\begin{array}{c}\text { Renormalization } \\
\text { redshift }(\mathrm{meV})\end{array}$ & $\begin{array}{c}\text { Screening } \\
\text { blueshift }(\mathrm{meV})\end{array}$ \\
\hline $\mathrm{ZnO}$ & $5 \times 10^{15}$ & 4.5 & 2.1 \\
$\mathrm{GaN}$ & $5 \times 10^{15}$ & 3.5 & 3.6 \\
$\mathrm{ZnO}$ & $1 \times 10^{16}$ & 5.7 & 2.7 \\
$\mathrm{GaN}$ & $1 \times 10^{16}$ & 4.5 & 4.5 \\
$\mathrm{ZnO}$ & $5 \times 10^{16}$ & 9.0 & 4.6 \\
$\mathrm{GaN}$ & $5 \times 10^{16}$ & 7.4 & 7.7 \\
\hline \hline
\end{tabular}

in the energy positions of the exciton transitions would be expected. These predictions are verified in Figs. 1-3.

These results show that the energy positions of optical transitions are modified when additional free electrons are introduced into the system. The energy positions are determined by the combined effects of screening and band gap renormalization resulting from the additional free electrons. This study establishes trends for the optical transition energies, but is not intended to give the exact energy positions. There are a number of unknowns, such as the exact number of free electrons introduced. Likewise we have not included the effect that holes will have on either screening or band gap renormalization. The holes will likely have a much shorter lifetime than the electrons and therefore their contribution should be significantly smaller than that of the electrons. We have not included temperature effects; however the samples are immersed in liquid $\mathrm{He}$ so these effects should not be great. In view of these factors, we believe the major contributors explain the experimental results.

In conclusion we have shown that the increased number of free electrons excited by the $\mathrm{Ar}^{+}$ion laser results in a combination of screening and band gap renormalization. The screening processes produce a blueshift of the optical transitions in both $\mathrm{GaN}$ and $\mathrm{ZnO}$. The increased number of free electrons also produce many-body effects resulting in the renormalization of the band gap. The renormalization lowers the band gap energy, producing a redshift of the optical transitions in both $\mathrm{GaN}$ and $\mathrm{ZnO}$. The combined effect of screening and renormalization produced a redshift of the optical transitions in $\mathrm{ZnO}$ and very little shift of the optical transitions in $\mathrm{GaN}$.

\section{ACKNOWLEDGMENTS}

The authors would like to thank C. Huang for technical support. The work of D.C.R., D.C.L., and B.J. was performed at Wright-Patterson Air Force Base under USAF Contract No. F33615-95-C-1619. This work was partially supported by the Air Force Office of Scientific Research (AFOSR).

\footnotetext{
${ }^{1}$ H. Schweitzer, A. Forchel, H. Hangleiter, S. Schmitt-Rink, J. P. Lowenau, and H. Huag, Phys. Rev. Lett. 51, 698 (1983).

${ }^{2}$ I. Balslev, Phys. Rev. B 30, 3203 (1984).

${ }^{3}$ S. K. Chun, J. Appl. Phys. 80, 4773 (1996).

${ }^{4}$ A. M. Bakiev, Yu V. Vandyshev, G. S. Volkov, V. S. Dneprovskii, Z. D. Kovalyuk, A. R. Lesiv, S. V. Savinov, and A. I. Furtichev, Sov. Phys. Solid State 28, 579 (1986).
} 
${ }^{5}$ C. Guillemot, Phys. Rev. B 31, 1428 (1985).

${ }^{6}$ J. A. Brum, G. Bastard, and C. Guillemot, Phys. Rev. B 30, 905 (1984).

${ }^{7}$ D. C. Reynolds, B. Jogai, P. W. Yu, K. R. Evans, and C. E. Stutz, Phys. Rev. B 46, 15274 (1992).

${ }^{8}$ K. A. Dunn, S. E. Babcock, R. Vaudo, V. Phanse, and J. Redwing, Mater. Res. Soc. Symp. Proc. 482, 417 (1998).

${ }^{9}$ D. C. Look, D. C. Reynolds, J. W. Hemsky, R. L. Jones, and J. R. Sizelove, Appl. Phys. Lett. 75, 811 (1999).
${ }^{10}$ D. C. Look, Electrical Characterization of GaAs Materials and Devices (Wiley, New York, 1989).

${ }^{11}$ P. Perlin et al., Appl. Phys. Lett. 68, 1114 (1996).

${ }^{12}$ B. K. Meyer, D. Volm, A. Graber, H. C. Alt, T. Detchprohm, A. Amano, and I. Akasaki, Solid State Commun. 95, 597 (1995).

${ }^{13}$ J. R. Haynes, Phys. Rev. Lett. 4, 361 (1960).

${ }^{14}$ L. Hedin and B. I. Lundquist, J. Phys. C 4, 2064 (1971).

${ }^{15}$ O. Gunnavson and B. I. Lundquist, Phys. Rev. B 13, 4274 (1976). 